\title{
Stimulated Absorption of Light in Bosonic Cascades of Excitons
}

\author{
Alexey Kavokin $1,2,3,4$ \\ Stella Kutrovskaya $a^{2,5^{*}}$ \\ Giuseppe Eramo ${ }^{6}$ \\ Tran D. Phong? \\ Nguyen Tran Thuat ${ }^{8}$
}

${ }^{1}$ CNR-SPIN, Viale del Politecnico 1, I-00133, Rome, Italy

${ }^{2}$ Russian Quantum Center, 143025 Skolkovo, Moscow region, Russia

${ }^{3}$ Spin Optics Laboratory, St-Petersburg State University, 1, Ulianovskaya, 198504, St-Petersburg, Russia

${ }^{4}$ Physics and Astronomy, University of Southampton, Highfield, Southampton, SO171BJ, United Kingdom

${ }^{5}$ A.G and N.G. Stoletov Vladimir State University (VSU), 87 Gorki st., Vladimir, 600000 Russia

${ }^{6}$ Mediterranean Institute of Fundamental Physics, 31, Appia Nuova, 00031, Rome, Italy

${ }^{7}$ University of Science and Technology of Hanoi, Vietnam Academy of Science, 18 Hoang Quoc Viet, Cau Giay District, Hanoi, Viet Nam

${ }^{8}$ Nano and Energy Center, VNU University of Science, 334 Nguyen Trai street, Thanh Xuan, Hanoi, Viet Nam

*Corresponding author.Tel.: +7915 76999 85.E-mail address: 11stella@mail.ru.

Keywords: stimulated absorption, bosonic condensates, microcavity.

Abstract:

In bosonic cascades of excitons or exciton-polaritons the absorption of electromagnetic radiation resonant to the energy spacing between neighboring quantization levels may be stimulated by the initial state occupation number. This opens way to realization of ultrasensitive detectors of infrared or terahertz radiation. The stimulated absorption of light by bosonic condensates has also a high potentiality for photovoltaic applications.

\section{Introduction}

The physics of bosonic condensates of superposition light-matter quasiparticles develops very rapidly in the recent decade. Polariton lasers based on non-equilibrium bosonic condensates of excitonpolaritons have been realised in various semiconductor systems including GaAs [1], CdTe [2], GaN [3], $\mathrm{ZnO}$ [4] based microcavities, $\mathrm{ZnO}$ microwires [5], organic microcavities [6]. In wide-band gap semiconductors, $\mathrm{GaN}$ and $\mathrm{ZnO}$, as well as in organic structures, polariton lasing has been observed at room temperature [3-6]. Moreover, the polariton lasers with electronic injection are currently being realised in $\mathrm{GaAs}$ [7] and $\mathrm{GaN}[8]$ microcavities.

In this context, the perspective of application of polariton devices in opto-electronics seems realistic. On the other hand, polariton devices still need to find their niche and demonstrate their advantages over conventional semiconductor lasers. Recently, the concept of bosonic cascades has been put forward $[9,10]$. A bosonic cascade represents a potential trap where bosonic quasiparticles (excitonpolaritons) are quantised (Fig. 1). Due to the essential non-equilibrium nature of polariton lasers, the formation of multiple bosonic condensates occupying different energy levels in the same potential trap is indeed possible. Multimode polariton lasing in parabolic potential traps has been experimentally demonstrated by Tosi et al. [11]

While the experimental effort towards demonstration of terahertz lasing in bosonic cascades is currently under way [12], we would like to propose an alternative application of bosonic cascades, that is not less pertinent, in our opinion.

\section{The concept of bosonic photodetectors}


Here we propose using the bosonic cascades of exciton-polaritons as ultrasensitive detectors of terahertz or infrared radiation. The concept is based on the stimulated absorption phenomenon characteristic of bosonic systems. Note, that the perspective of stimulated absorption in microcavities has been addressed in Ref 13 and studies experimentally in Ref. 14. However, the concept of high-sensitivity photodetectors based on polariton condensates has not been discussed yet, to the best of our knowledge. This concept is extremely simple. Consider an optical transition between a bosonic condensate characterised by the energy $E_{1}$ and the occupation number $N_{1}$ and another bosonic condensate characterised by the energy $E_{2}$ and the occupation number $N_{2}$. The probability to absorb an optical quantum of energy

is dependent on both occupation numbers:

$$
E=E_{2}-E_{1}
$$

$$
A \sim V N_{1}\left(N_{2}+1\right) \text {. }
$$

Here $V$ is the matrix element of a dipole operator for the optical transition between the energy levels $E_{1}$ and $E_{2}$. The probability of emission of the same optical quantum is

Straightforwardly, the net absorption is

$$
I \sim V N_{2}\left(N_{1}+1\right) \text {. }
$$

$$
A-I \sim V\left(N_{1}-N_{2}\right) \text {. }
$$

Having in mind that the difference $\left(N_{1}-N_{2}\right)$ is controllable by pumping strength and geometry, and it can easily achieve $10^{4}-10^{5}$ is realistic microcavity devices, the probability of resonant absorption of light in a two-level bosonic system is already quite high. Experimentally, the enhanced terahertz absorption in the presence of a bosonic condensates of exciton-polaritons in a microcavity has been studied in Ref. 14, which has also put forward this effect as a tool for pumping optically dark exciton states.

Here we propose to take a supplementary advantage of the cascade effect. Let us consider a system of $m$ equidistant quantum levels occupied by bosonic condensates of exciton-polaritons with occupation numbers $N_{1}, N_{2}, \ldots, N_{\mathrm{m}}$, respectively. Assuming that the dipole matrix elements of optical transitions between any pair of neighbouring states in the cascade are all equal, we end up with the net absorption probability of

$$
A-I \sim V\left(N_{1}-N_{\mathrm{m}}\right) \text {. }
$$

Keeping the highest energy level in the cascade empty or nearly empty, we achieve the net absorption of

$$
A_{\max } \sim V N_{1} \text {. }
$$

The absorption of terahertz photons may have a peculiar effect on the set of occupation numbers $N_{1}, N_{2}, \ldots$ , $N_{\mathrm{m}}$. This effect may be conveniently analysed using the system of rate equations for the polariton occupation numbers having a generic form:

$$
\frac{d N_{i}}{d t}=V\left(N_{\mathrm{i}-1}-N_{\mathrm{i}+1}\right) N_{\mathrm{THz}}-\frac{N_{i}}{\tau}+P_{\mathrm{i}}
$$

where $\tau$ is the life-time (radiative or non-radiative) of an exciton-polariton in a trap, $P_{\mathrm{i}}$ is the external pumping term, $N_{\mathrm{THz}}$ is the number of terahertz (infrared) photons arriving to the trap at the time $t$. For $\mathrm{i}=1$ the first term in the right part of Eq. (7) changes to $V\left(N_{1}-N_{2}\right)$. To detect the terahertz (infrared) absorption one should be measuring the optical emission intensity by the polariton condensates $N_{1}, N_{2}, \ldots$, $N_{\mathrm{m}}$. Clearly, one can tune the sensitivity of the photodetection by the cascade by tuning the external pumping strength described by the coefficients $P_{\mathrm{i}}$. Moreover, the detection frequency may be scanned by tuning the curvature of the parabolic trap and changing the interlevel spacing in the cascade. This may be realised e.g. in the electrostatic traps similar to ones studied in Ref. 15. 

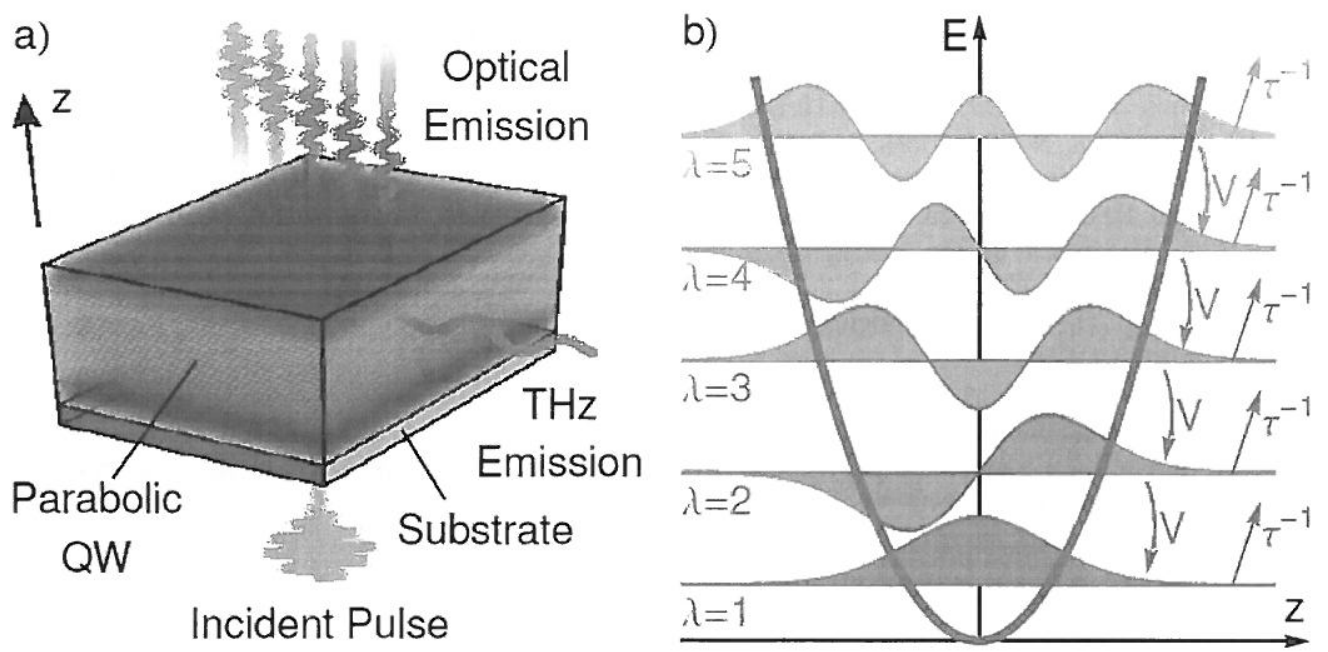

Fig. 1. The schematic of a bosonic cascade lasers proposed in a series of theoretical works $[9,10]$. The planar microcavity structure with an embedded parabolic quantum well would emit light in the optical frequency range in the normal to plane direction (a) and it would emit the terahertz radiation in the plane of the structure (a). The sequence of energy levels occupied by bosonic condensates of exciton-polaritons in a parabolic quantum well potential is shown in the panel (b). The polaritons can either radiatively decay with a rate $\tau^{-1}$ or relax in energy to the level below emitting a terahertz photon, with a rate $V$.

In conclusion, the goal of this short communication is in attracting the attention of the community to the potentiality of polariton based bosonic systems for the ultra-sensitive detection of infrared or terahertz quanta. We believe that the effect of bosonic stimulation of absorption may be of a significant importance for studies of extremely weak electromagnetic signals coming e.g. from distant stars. In what concerns the photovoltaic applications, the limitation imposed by the narrow band absorption in each trap may be compensated by the use of multiple traps of different depths that would cover a sufficiently large frequency range. Combined with conventional silicon based photocells, the photocells based on stimulated absorption of light in traps may lead to the enhanced quantum efficiency.

Acknowledgement. This research has been supported by the Ministry of Education and Science of the Russian Federation (state project no. 16.1123.2017/PCH), RFBR grants16-32-60067 mol_a_dk. AK acknowledges the support from the EPSRC Programme grant in Hybrid Polaritonics.

\section{References:}

[1] D. Bajoni, P. Senellart, E. Wertz, I. Sagnes, A. Miard, A. Lema^ttre, and J. Bloch, Polariton Laser Using Single Micropillar GaAs-GaAlAs Semiconductor Cavities, Phys. Rev. Lett., 2008, 100, DOI: 10.1103/PhysRevLett.100.047401.

[2] J. Kasprzak, M. Richard, S. Kundermann, A. Baas, P. Jeambrun, J. M. J. Keeling, F. M. Marchetti, M. H. Szyman'nska, R. Andr'e, J. L. Staehli, V. Savona, P. B. Littlewood, B. Deveaud, and Le Si Dang, Bose-Einstein condensation of excitonpolaritons, Nature, 2006, 443, 2006, DOI:10.1038/nature05131.

[3] S. Christopoulos, G. Baldassarri H"oger von H"ogersthal, A. J. D. Grundy, P. G. Lagoudakis, A. V. Kavokin, J. J. Baumberg, G. Christmann, R. Butt'e, E. Feltin, J.-F. Carlin, and N. Grandjean, Room-Temperature Polariton Lasing in Semiconductor Microcavities, Phys. Rev. Lett., 2007, 98, DOI:10.1103/PhysRevLett.98.126405.

[4] F. Li, L. Orosz, O. Kamoun, S. Bouchoule, C. Brimont, P. Disseix, T. Guillet, X. Lafosse, M. Leroux, J. Leymarie, M. Mexis, M. Mihailovic, G. Patriarche, F. Reveret, D. Solnyshkov, J. Zuniga-Perez, and G. Malpuech, From Excitonic to Photonic Polariton Condensate in a ZnOBased Microcavity, Phys. Rev. Lett., 2013, 110, DOI:10.1103/PhysRevLett.110.196406.

[5] W. Xie, H. Dong, S. Zhang, L. Sun, W. Zhou, Y Ling, J. Lu, X. Shen, and Z. Chen, Room-Temperature Polariton Parametric Scattering Driven by a One-Dimensional Polariton Condensate, Phys. Rev. Lett., 2012, 108, DOI:10.1103/PhysRevLett.108.166401.

[6] K. S. Daskalis, S. A. Maier, R. Murray, and S. Kena-Cohen, Nonlinear interactions in an organic polariton condensate, Nature Mater., 2014, 13, DOI:10.1038/nmat3874.

[7] C. Schneider, A. Rahimi-Iman, N. Y. Kim, J. Fischer, I. G. Savenko, M. Amthor, M. Lermer, A. Wolf, L. Worschech, V. D. Kulakovskii, I. A. Shelykh, M. Kamp, S. Reitzenstein, A. Forchel, Y. Yamamoto, and S. H"ofling, An electrically pumped polariton laser, Nature, 2013, 497, DOI:10.1038/nature12036.

[8] P. Bhattacharya, T. Frost, S. Deshpande, M. Z. Baten, A. Hazari, and A. Das, Room Temperature Electrically Injected Polariton Laser, Phys. Rev. Lett., 2014, 112, DOI:10.1103/PhysRevLett.112.236802. 
[9] T. C. H. Liew, M. M. Glazov, K. V. Kavokin, I. A. Shelykh, M. A. Kaliteevski, and A. V. Kavokin, Proposal for a Bosonic Cascade Laser, Phys. Rev. Lett., 2013, 110, DOI:10.1103/PhysRevLett.110.047402.

[10] M. A. Kaliteevski, K. A. Ivanov, G. Pozina, and A. J. Gallant, Single and double bosonic stimulation of THz emission in polaritonic systems, Sci. Rep., 2014, 4, DOI:10.1038/srep05444.

[11] G. Tosi, G. Christmann, N. G. Berloff, P. Tsotsis, T. Gao, Z. Hatzopoulos, P. G. Savvidis, and J. J. Baumberg, Sculpting oscillators with light within a nonlinear quantum fluid, Nature Phys., 2012, 8, DOI:10.1038/nphys2182.

[12] A. V. Trifonov, E. D. Cherotchenko, J. L. Carthy, I. V. Ignatiev, A. Tzimis, S. Tsintzos, Z. Hatzopoulos, P. G. Savvidis, and A. V. Kavokin, Dynamics of the energy relaxation in a parabolic quantum well laser, Phys. Rev. B, 2016, 93, DOI:10.1103/PhysRevB.93.125304.

[13] A.V. Kavokin and G. Eramo, Photocurrents induced by stimulated absorption of light, Natural Science, 2010, 2, DOI:10.4236/ns.2010.21010.

[14] J.-M. Me'nard, C. Poellmann, M. Porer, U. Leierseder, E. Galopin, A. Lemâ^tre, A. Amo, J. Bloch \& R. Huber, Revealing the dark side of a bright exciton-polariton condensate, Nature Communications, 2014, 5, DOI:10.1038/ncomms5648.

[15] R. Balili, V. Hartwell, D. Snoke, and K. West, Bose-Einstein Condensation of Microcavity Polaritons in a Trap, Science 2007, 316, DOI:10.1126/science. 1140990. 